\title{
Clinical Observations on Treatment for Chinese Patients with Gastrointestinal Stromal Tumors
}

\author{
Zhan-De He ${ }^{1}$, Xin-En Huang ${ }^{2}$, Jian-Nong Zhou ${ }^{3 *}$
}

\begin{abstract}
Background: To investigate the diagnostic and treatment methods for Chinese patients with gastrointestinal stromal tumor (GIST). Materials and Methods: From January 2004 to June 2014, patients diagnosed with primary GIST and treated by a single medical team in the Department of Digestive Disease of XuYi Hospital of Traditional Chinese Medicine were retrospectively recruited. Re-examination and follow-up was conducted regularly and abdominal enhanced CT, blood biochemistry and responses to surgery or imatinib were recorded. Results: A total of 15 patients were enrolled, including 9 male and 6 female patients, with an average age of 54 years (ranging from 32-81 years). The primary symptoms were abdominal uncomfortable in 5 patients, abdominal pain in 6 patients as well as nausea and vomiting in 4 patients. One patient was diagnosed with bowl obstruction at the first visit. All patients were treated with surgery, and tumor site was confirmed 1 esophagus, 6 stomach, 4 small bowel, and 4 colorectal and all patients were pathologically diagnosed with GIST. Immunochemical test positive for CD 117 was found 12 patients, and positive for CD 34 in 7 patients. The median follow-up time was 24 months (range of 3-63). Three metastasis were confirmed 1.5, 2 and 2.6 years postoperatively. Three patients were treatment by imatinib postoperatively. Conclusions: Surgery remains the main treatment method for Chinese patients with GIST and imatinib could be feasible and safe for treating Chinese patients with GIST.
\end{abstract}

Keywords: Gastrointestinal stromal tumor - imatinib mesylate - postoperative therapy

Asian Pac J Cancer Prev, 16 (12), 5111-5113

\section{Introduction}

At present, a so called guideline based treatment for patients with gastrointestinal stromal tumor (GIST) is radical surgery with a purpose to achieve a negative microscopic margins (Hohenberger et al., 2010). After this, the concerning is focused on GIST patients who are at risk of recurrence and it is estimated that nearly 40$50 \%$ of patients who had radical resections is supposed to develop recurrence or metastasis (Rutkowshi et al., 2008). For some cases with specific anatomic locations, e.g., esophagogastric junction, duodenum, or low rectum, as well as with huge tumor diameters, the tumor cannot be completely resected (Corless et al., 2004; Bosman et al., 2010). On this occasion, treatment for these patients could be dangerous if extensive procedures are performed, and postoperative complications and mortality could be high.

In recent years, due to better understanding of the molecular pathogenesis of GIST, imatinib mesylate is considered as a targeted therapy with clinical effectiveness for nonsurgical treatment for GIST, and was approved for clinical use in many countries. Previously published research suggested that imatinib considerably improves the prognosis of patients with locally advanced and metastatic GIST (Joensuu et al., 2012; Joensuu et al., 2013). A clinical trial with a large sample size demonstrated a median time to progression of 24 months and a median overall survival time of 57 months focusing on treatment with imatinib for patients with unresectable or metastatic GIST (Blanke et al., 2008). Furthermore, recent reports suggested that preoperative imatinib use can increase the resectability rate and reduce procedure-related mortality, thereby improving the survival of GIST patients (Rutkowski et al., 2013; Wang et al., 2013). But it is not clear in China, what kind of combination of treatment, especially, with imatinib could be recommended.

On this background, the aim of this study was to retrospectivelly analyze the clinical efficacy of an institional treatment for Chinese patients with unresectable or metastatic/recurrent GIST, especially with imatinib.

\section{Materials and Methods}

\section{Patient selection}

From January 2004 to June 2014, patients diagnosed with primary GIST and treated by a single medical team in the Department of Digestive Disease of XuYi Hospital of Traditional Chinese Medicine were retrospectively recruited. Re-examination and follow-up was conducted regularly and abdominal enhanced CT, blood biochemistry and responses to surgery or imatinib were recorded.The inclusion criteria were as follows: $i$ ) patients with primarily

${ }^{1}$ Department of Digestive Disease, XuYi Hospital of Traditional Chinese Medicine, Jiangsu, ${ }^{2}$ Department of Medical Oncology, ${ }^{3}$ Department of Colorectal Cancer, Jiangsu Cancer Hospital, Nanjing, China *For correspondence: huangxinen06@126.com 
resectable or metastatic/recurrent GIST (unresectable tumors were defined as being huge in size or having already infiltrated the adjacent tissues and organs with unclear boundaries, according to radiological findings); ii) GIST that was confirmed by immunohistochemical staining and/or KIT and platelet-derived growth factor receptor A (PDGFRA) mutation detection iii) patients with no history of taking imatinib and all patients were age more than 18 years. The exclusion criteria were as follows: i) patients with contraindications to imatinib therapy; $i$ ) pregnant or lactating women; and iii) diagnosed with concurrent malignant tumors.

\section{Surgery and imatinib treatment}

All of the patients signed informed consent before treatment. Imatinib was administered at a dosage of 400 $\mathrm{mg} / \mathrm{d}$. Clinical efficacy and drug toxicity were assessed after two cycles of treatment if imatinib was used. Patient responses were evaluated according to the Choi criteria 11.

All surgical procedures were conducted by the same medical team at the XuYi Hospital. The primary surgical aims were to maintain as many residual organ functions as possible and to achieve R0 resection as frequently as possible. To achieve a radical cure, additional debulking surgery was performed in some cases, mainly if the tumor had infiltrated adjacent tissues and organs. Frozen slices of the incisal margin and tumor were routinely collected during surgery.

\section{Follow-up of patient}

All included patients were mainly followed up on an inpatient and/or outpatient basis or through telephone call. The follow-up evaluation included abdominal CT, whole blood assessment and classification, and evaluations on liver and kidney functions.

\section{Results}

A total of 15 patients were enrolled, including 9 male and 6 female patients, with an average age of 54 years (ranging from 32-81 years). The primary symptoms were abdominal uncomfortable in 5 patients, abdominal pain in 6 patients as well as nausea and vomiting in 4 patients. One patient was diagnosed with bowl obstruction at the first visit. All patients were treated with surgery, and tumor site was confirmed 1 esophagus, 6 stomach, 4 small bowel, and 4 colorectal and all patients were pathologically diagnosed with GIST. Immunochemical test positive for CD 117 was found 12 patients, and positive for CD 34 in 7 patients. The median follow-up time was 24 months (range of 3-63). Three metastasis were confirmed 1.5, 2 and 2.6 years postoperatively. Three patients were treatment by imatinib postoperatively.

\section{Discussion}

The diagnosis of GIST during these 20 years has demonstrated an increased trend, and an important reason was suggested partially due to a modification of diagnostic reclassification and guidelines (Perez et al., 2006). And now, it is reported that GIST could be considered as a most common mesenchymal tumor of the digestive tract (Joensuu et al., 2013), that is associated with an origination from a common precursor of the interstitial Cajal cells or its precursor cells characterized by the presence of activating mutations in KIT and platelet-derived growth factor receptor (PDGFR) (Cassier et al., 2010; Liegl-Atzwanger et al., 2010). For patients with localized primary GIST, surgical resection with curative intent is still a mainstay of therapy. However, after complete surgery, the risk of relapse is estimated approximately $40 \%$, with multiple variations based on known clinicopathologic features. Two clinical referred classification systems, the Fletcher and Miettinen, consisting of prognostic factors, e.g., primary site, size, and mitotic index, are used to stratify patients into groups from low-, intermediate-, to high-risk of recurrence. (Fletcher et al., 2002; Miettinen et al., 2006). During these years, one important progress regarding the treatment of GIST is the clinical administration of imatinib , which was originally designed as an inhibitor of Bcr-Abl but was later demonstrated to be effective in inhibiting tyrosine kinase activities of KIT and PDGFR, and it is demonstrated that imatinib significantly improves survival for patients with advanced GIST, and it has become a standard of care in this setting (Demetri et al., 2002; Blanke et al., 2008). Furthermore, adjuvant imatinib administered in high-risk patients for 12 months after surgical removal of GIST with Kit protein expression is associated with prolong recurrence-free survival compared to placebo (Nilsson et al., 2007; Dematteo et al., 2009). Based on these results, imatinib was approved at a daily dose of $400 \mathrm{mg}$ by the US Food and Drug Administration (FDA) and the European Medicine Agency (EMA) in 2008 and 2009, respectively, as adjuvant therapy for high-risk patients following complete surgical resection of GIST. Recently, a randomized Phase III trial, which included 400 high-risk GIST patients, reported a statistically significant improvement of RFS (65.6\% vs $47.9 \%)$ and overall survival (OS) (92\% vs $81.7 \%$ ) after 3 years of imatinib as opposed to 1 year (Joensuu et al., 2012). Following these results, the FDA and EMA recommended 36 months of adjuvant treatment in high-risk patients, simultaneously noting that the optimal duration of therapy remains to be established. Translational studies indicated variability in the response to imatinib therapy according to molecular and genetic characteristics of GIST, including the cKIT (exons 9,11,13, and 17) and the PDGFRA gene (exons 12, 14, and 18), allowing thus tailored therapy. Specifically, in the advanced stage, the recommended doses include imatinib $400 \mathrm{mg}$ (mutations in cKIT exon 11, PDGFRA non-D842V, cKIT other sites, BRAF, NF1, NRAS/KRAS, SDHB/C, and IGF1R overexpression), $800 \mathrm{mg}$ (mutations in exon 9), and no treatment (in PDGFRA D842V) (Blay et al., 2012; ESMO/European et al.,2012). When adjuvant therapy is required, imatinib $400 \mathrm{mg}$ is recommended for mutations in cKIT exon 11 or 9, in PDGFRA non-D842V, and in cKIT other sites but no therapy in the remaining subgroups (Blay et al., 2012; ESMO/European et al., 2012).

In current study, from January 2004 to June 2014, we recruited a total of 15 patients that included 9 male and 6 female patients, with an average age of 54 years 
(ranging from 32-81 years). The primary symptoms were abdominal uncomfortable in 5 patients, abdominal pain in 6 patients as well as nausea and vomiting in 4 patients. One patient was diagnosed with bowl obstruction at the first visit. All patients were treated with surgery, and tumor site was confirmed 1 esophagus, 6 stomach, 4 small bowel, and 4 colorectal and all patients were pathologically diagnosed with GIST. Immunochemical test positive for CD 117 was found 12 patients, and positive for CD 34 in7 patients. The median follow-up time was 24 months (range of 3-63). Three metastasis were confirmed 1.5, 2 and 2.6 years postoperatively. Three patients were treatment by imatinib postoperatively. And the treatment related death was not reported in our current study. In conclusion, we suggested that surgery remains the main treatment method for Chinese patients with GIST in rural area and imatinib could be feasible and safe for treating Chinese patients with GIST.

In conclusion, application of imatinib as a preoperative therapy is feasible and safe for primarily unresectable or metastatic/recurrent GISTs in Chinese patients and this treatment can effectively decrease tumor size, facilitating successful tumor resection.

\section{References}

Blanke CD, Demetri GD, von Mehren M, et al (2008). Long-term results from a randomized phase II trial of standard-versus higher-dose imatinib mesylate for patients with unresectable or metastastic gastrointestinal stromal tumors expressing KIT. J Clin Oncol, 26, 620-5.

Blanke CD, Rankin C, Demetri GD, et al (2008). Phase III randomized, intergroup trial assessing imatinib mesylate at two dose levels in patients with unresectable or metastatic gastrointestinal stromal tumors expressing the kit receptor tyrosine kinase: S0033. J Clin Oncol, 26, 626-32.

Blay JY, Le Cesne A, Cassier PA, Ray-Coquard IL (2012). Gastrointestinal stromal tumors (GIST): a rare entity, a tumor model for personalized therapy, and yet ten different molecular subtypes. Discov Med, 13, 357-367.

Bosman F T, Carneiro F, Hruban R H (2010). WHO classification of tumours of the digestive system 4th ed.: IARC, Lyon Press. 74-6.6(ed).

Cassier PA, Ducimetie're F, Lurkin A, et al (2010). A prospective epidemiological study of new incident GISTs during two consecutive years in Rh\&ocirc;ne Alpes region: incidence and molecular distribution of GIST in a European region. Br J Cancer, 103, 165-70.

Corless CL, Feltcher JA, Heinrich MC (2004). Biology of gastrointestinal Stromal tumors. J Clin Oncol, 22, 3813-25.

Dematteo RP, Ballman KV, Antonescu CR, et al (2009). American college of surgeons oncology group (ACOSOG) intergroup adjuvant GIST study team adjuvant imatinib mesylate after resection of localised, primary gastrointestinal stromal tumour: a randomised, double-blind, placebocontrolled trial. Lancet, 373, 1097-104.

Demetri GD, von Mehren M, Blanke CD, et al (2002). Efficacy and safety of imatinib mesylate in advanced gastrointestinal stromal tumors. N Engl J Med, 347, 472-80.

ESMO/European Sarcoma Network Working Group Gastrointestinal stromal tumors (2012): ESMO Clinical Practice Guidelines for diagnosis, treatment and follow-up. Ann Oncol, 23, 49-55.

Fletcher CD, Berman JJ, Corless C, et al (2002). Diagnosis of gastrointestinal stromal tumors: a consensus approach. Int J Surg Pathol, 10, 81-9.

Hohenberger P, Eisenberg B (2010). Role of surgery combined with kinase inhibition in management of gastrointestinal Stromal tumor (GIST). Ann Surg Oncol, 17, 2585-600.

Joensuu H, Eriksson M, Sundby Hall K, et al (2012). One vs three years of adjuvant imatinib for operable gastrointestinal stromal tumor a randomized trial 2012.JAMA, 307, 1265-72.

Joensuu H, Hohenberger P, Corless CL (2013). Gastrointestinal stromal tumour. Lancet, 382, 973-83.

Liegl-Atzwanger B, Fletcher JA, Flethcher CD (2010). Gastrointestinal stromal tumors.Virchows Arch, 456, 111-27.

Miettinen M, Lasota J (2006). Gastrointestinal stromal tumors: pathology and prognosis at different sites. Semin Diagn Pathol, 23, 70-83.

Nilsson B, Sjolund K, Kindblom LG, et al (2007). Adjuvant imatinib treatment improves recurrence-free survival in patients with high-risk gastrointestinal stromal tumours (GIST). Br J Cancer, 96, 1656-8.

Perez EA, Livingstone AS, Franceschi D, et al (2006).Current incidence and outcomes of gastrointestinal mesenchymal tumors including gastrointestinal stromal tumors. J Am Coll Surg, 202, 623-9.

Rutkowshi P,Debiec-Rychter M, Ruka W (2008). Gastrointestinal stromal tumors: key to diagnosis and choice of therapy. Mol Diagn Ther, 12, 131-43.

Rutkowski P, Gronchi A, Hohenberger P, et al (2013). Neoadjuvant imatinib in locally advanced gastrointestinal stromal tumors (GIST): the EORTC STBSG experience. Ann Surg Oncol, 20, 2937-43.

Wang C, Zheng B, Chen Y, et al (2013). Imatinib as preoperative therapy in Chinese patients with recurrent or metastatic GISTs. Chin J Cancer Res, 25, 63-70. 